\title{
AVALIAÇÃO DOS FATORES RELACIONADOS A PREVALENCIA DA DESNUTRIÇÃO EM CRIANÇAS YANOMAMIS MENORES DE CINCO ANOS DE IDADE EM RORAIMA
}

Eliene Mendes de OLIVEIRA*

Helenilson José Soares BONIARES**

Ricardo Alves da FONSECA***

\begin{abstract}
* Aluna do Mestrado em Ciências da Saúde da Universidade Federal de Roraima.Enfermeira, Especialista em Saúde Coletiva com Ênfase para Saúde Indígena, Professora do curso de Enfermagem da Faculdade Roraimense de Ensino Superior. E-mail: mendeseliene@ hotmail.com

**Aluno do Mestrado em Ciências da Saúde da Universidade Federal de Roraima.Farmacêutico, Especialista em

Farmacologia Clinica, Especialista em Gestão da Assistência farmacêutica. E-mail:helenilsonb@gmail.com
\end{abstract}

Dr. Ricardo Alves da Fonseca

Prof. do Mestrado em Ciências da Saúde da Universidade Federal de Roraima

Recebido em: 30/08/2015 - Aprovado em: 04/05/2016 - Disponibilizado em: 30/07/2016

\begin{abstract}
Resumo: O estudo objetivou conhecer a situação nutricional das crianças indígenas do Polo Base Haxiu, DseiYanomami, Estado de Roraima; com base em dados fornecidos pela Secretaria Especial de Saúde Indígena (SESAI), referente ao ano de 2014. A pesquisa foi do tipo documental e caráter quantitativo a qual contou com dados fornecidos pela Secretaria Especial de Saúde Indígena (SESAI). Foi avaliada a desnutrição de crianças em duas faixas etárias: de zero a doisanos e de zero a cinco anos de idade. O estudo apontou para a alarmante situação a que estão expostas as crianças indígenas do polo base Haxiu no Distrito Especial Indígena Yanomami em Roraima. Conclui-se que o estado nutricional das crianças do Polo Base Haxiu, Dsei Yanomami encontra-se em situação de alerta, portanto, faz-se necessário, medidas que visem à importância da prevenção da desnutrição infantil, seja ela primária ou secundária, entre as crianças indígenas.
\end{abstract}

Palavras-chave: Desnutrição Infantil. Criança Indígena. Yanomami.

\begin{abstract}
Undernutrition constitutes a public health problem and developing countries, like Brazil, are the most affected. Health conditions such as food, sanitation, scarcity of resources and a focused work for health education are fully connected with the problem. Among the indigenous population, where these problems are most relevant, under nutrition appears as a major cause of infant mortality. In this way, the indigenous population must have a differentiated attention. The study aimed to analyze the prevalence of indigenous child under nutrition aged between 0 to 5 years old, in communities served by the Yanomami district, Auar is pole based, at Amajari municipality, in the State of Roraima, in 2014; and the factors that are linked to the events. The research was quantitative descriptive of which included data provided by the "Secretaria Especial de Saúde Indígena (SESAI)". The under nutrition of children was evaluated in two age groups: $0-2$ years and $0-5$ years old. The study pointed to the alarming situation they are exposed the indigenous children in the community in Roraima.
\end{abstract}

Keywords: Under nutrition. Indigenous Children .Yanomami.

\section{Introdução}

A Desnutrição é um importante problema de saúde pública nos países em desenvolvimento, e pessoas mais pobres são as mais afetadas 200 milhões de crianças desnutridas em todo o mundo, estão entre as causas da desnutrição, sobretudo o desmame precoce ou tardio, introdução inadequada de alimentos complementares, a higiene precária no preparo dos alimentos, os hábitos alimentares inadequados, o déficit específico de micronutrientes, a alta frequência de infecções, em particular de doenças diarreicas e parasitoses intestinais, dentre outros (WAITZBERG, 2009). 
O Ministério da Saúde (BRASIL, 2005) afirma que a desnutrição pode começar precocemente na vida intra-uterina (baixo peso ao nascer) e frequentemente cedo na infância, em decorrênciada interrupção precoce do aleitamento materno exclusivo e da alimentação complementar inadequada nos primeiros dois anos de vida, associada, muitas vezes, à privação alimentar ao longo da vida e à ocorrência de repetidos episódios de doenças infecciosas (diarreicas e respiratórias). Isso gera a desnutrição primária. Outros fatores de risco na gênese da desnutrição incluem problemas familiares relacionados com a situação sócioeconômica,das mães sobre os cuidados com a criança pequena (alimentação, higiene e cuidados com a saúde de modo geral) e o fraco vínculo mãe e filho.

Segundo dados do IBGE (2009), se falando de saúde pública, o Brasil teve uma diminuição nos casos de mortalidade infantil, no entanto esses avanços alcançadosmostram diferenças entre as populações, podendo ser observado, quando comprados com as crianças indígenas, um exemplo é a taxa de mortalidade infantil, que para a população indígena é de 41,9 por mil nascidos vivos, enquanto a taxa nacional foi em torno de 19,0 por mil nascidos vivos, segundo dados do IBGE/Pnad (IBGE, 2010).Quanto à mortalidade infantil entre os anos de 2000 a 2009 foram registrados 6.754 óbitos em crianças menores de um ano de idade. Índice médio de mortalidade da criança indígena atéanos é quase que o dobro do índice médio de mortalidade da criança não indígena, lembrando que a desnutrição infantil aparece como a principal causa de mortalidade em crianças indígenas.

Nos últimos anos, a prevalência de desnutrição em crianças menores de cinco anos apresentou queda significativa, impactando na superação do Objetivo de Desenvolvimento do Milênio relacionado à redução da mortalidade da infância. No entanto, populações específicas e em situação de vulnerabilidade social, como indígenas e quilombolas, não acompanharam tal redução. Reafirma o que mostrou o IBGE, sobre as diferenças entre as populações indígenas para as não indígenas no quesito assistência à saúde.

O de Roraima por se encontrar dentro da Amazônia Legal Brasileira, na qual abriga grande parte da população indígena, os dados do censo de 2010 mostram que os dez municípios que apresentaram maior proporção de indígenas no total da população, nos últimos dez anos, onde apresentou o maior percentual, $88,1 \%$, foi o município de Uiramutã, localizado ao Nordeste de Roraima. Dos dez municípios, quatro encontram-se no Estado de Roraima. Alémde outros como, Normandia com (56,9\%), Pacaraima com $(55,4 \%)$ e Amajari com $(53,8 \%)$ fazem parte dos municípios com maior proporção de indígenas. ParaSecretaria do Índio (BRASIL, 
2014), existem mais de quinhentas comunidades indígenas no Estado.

Os índios Yanomamis constituem um conjunto cultural linguístico composto por quatro subgrupos territorialmente adjacentes que falam línguas mutuamente inteligíveis, porém(que compreende aproximadamente $56 \%$ da população), o Yanomami (ou Yanomae) (25\%), o Sanumá (14\%) e o Ninam (ou Yanam) (5\%).Pequena parte de seu território tradicional, situado no extremo noroeste de Roraima, é dividido com os vizinhos Ye'kuana ou Maiongong, pertencentes à família Karib(PORTAL G1, 2013).A sede do DSEI-Yanomami em Boa Vista, capital do estado de Roraima, é local onde são coordenadas todas as ações em saúde para atender os índios da etnia Yanomami. Na estrutura do DSEI-Yanomami, existem trinta e sete Pólos-Base, que operam como unidades básicas de saúde e prestam assistência a cerca de 19.000 indígenas das etnias Yanomamie Ye'kuana que residem em aproximadamente 272 aldeias, situadas entre os Estados do Amazonas e Roraima.

A presente pesquisa teve como objetivo, do Polo Base Haxiu, DseiYanomami, Estado de Roraima;dados fornecidos pela Secretaria Especial de Saúde Indígena (SESAI), referente ao ano de 2014.

\section{Métodos}

Foi realizada uma pesquisa do tipo documental e caráter quantitativobaseada em banco de dados do Distrito Sanitário Especial Indígena (Dsei Yanomami), onde foram selecionados dados sobre a desnutrição em crianças Yanomami, no período de janeiro a dezembro de 2014, nos polos base do Haxiu. que faz parte do Distrito Sanitário Especial Indígena Yanomami em Roraima, onde a desnutrição apresentou-se como uma dos principais agravo entre as crianças indígenas.

O polo base Наxiu localiza-se no Estado de Roraima no município de Alto Alegre,comuma população total de 896 pessoas distribuídas em 16 comunidades,o estudo optou por trabalhar os casos registrados apenas em 11comunidades, cuja população residente é de 705 pessoas, a delimitação ocorreu por falta de dados sobre as demais comunidades (PANTOJA, 2014). Entrea população ,130crianças menores de cinco anos de idade totalizando 18,4\%.O critério de inclusão utilizado na pesquisa foi o polo base com maior quantitativo de registros de desnutrição infantil sobre responsabilidade do DSEIYanomami. A coleta de dados foiDistrito Sanitário Especial Indígena/Secretaria Especial de Saúde Indígena de Roraima.

Os dados foram avaliados e em seguida feita uma análise para determinar se há a existência significativa de desnutrição nas comunidades,em seguida feita uma ligação do já se tem publicado sobre o tema, dentro da população indígena. 


\section{Resultados}

No ano de 2014, foram avaliadas $100 \%$ das crianças no polo base Haxiuonde possibilitou classificar o estado nutricional dessas crianças em muito baixo peso, baixo peso e peso adequado.

Observe a figura 1 que apresenta o percentual do estado nutricional de crianças com idade de zero a cinco anos de idade, no polo base Haxiu, no DESY Yanomami, Roraima.

Figura 1 - Percentual do estado nutricional de crianças com 0 - 2 anos de idade, no polo base Haxiu, no DESY Yanomami, RR

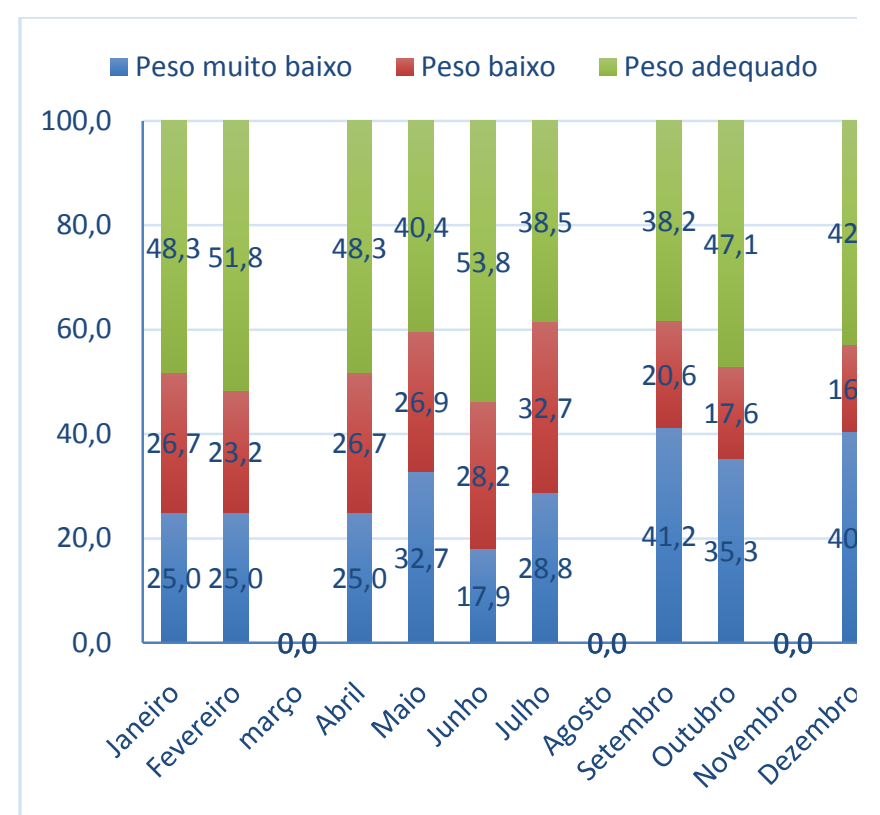

Fonte: Distrito Especial de Saúde Indígena-DSEY/ SESAI (2015).

Analisando a figura 1 podemos observar que,em janeiro de 2014 o percentual era de $25 \%$ para crianças com muito baixo peso, e em dezembro de 2014, considerando a mesma faixa etária, o percentual de crianças com muito baixo peso foi de 40,5\%; pode ser observado também uma redução de $10 \%$ dos casos de crianças com baixo peso e de aproximadamente $5,0 \%$ para crianças com peso adequado nos meses de janeiro em relação a dezembro de 2014.

Observe a figura 2 que apresenta o percentual do estado nutricional de crianças com 2 - 5 anos de idade, no polo base Haxiu, no DESY Yanomami, Roraima.

Figura 2 - Percentual do estado nutricional de crianças com 2 - 5 anos de idade, no polo base Haxiu, no DESY Yanomami, RR

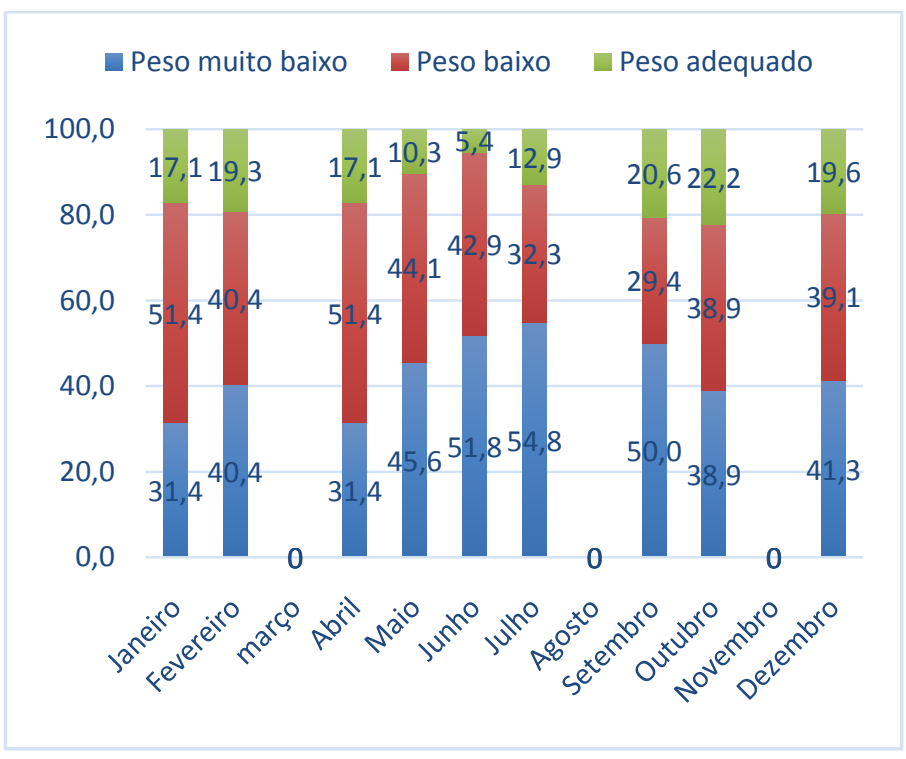

Fonte: Distrito Especial de Saúde Indígena-DSEY/ SESAI (2015).

Analisando a figura 2 podemos observar que, em janeiro de 2014 o percentual era de $31,4 \%$ para crianças com muito baixo peso e em dezembro de 2014, considerando a mesma faixa etária o percentual de crianças com muito baixo peso foi de $41,3 \%$; pode ser observado também uma redução de 12,3\% dos casos de crianças com baixo peso e de $2,5 \%$ para crianças com peso adequado nos meses de janeiro em relação a dezembro.

Observe a figura 3 que apresenta o percentual do estado nutricional de crianças com $0-5$ 
anos de idade, no polo base Haxiu, no DESY Yanomami, Roraima.

Figura 3 - Percentual do estado nutricional de crianças com $0-5$ anos de idade, no polo base Haxiu, no DESY Yanomami, RR

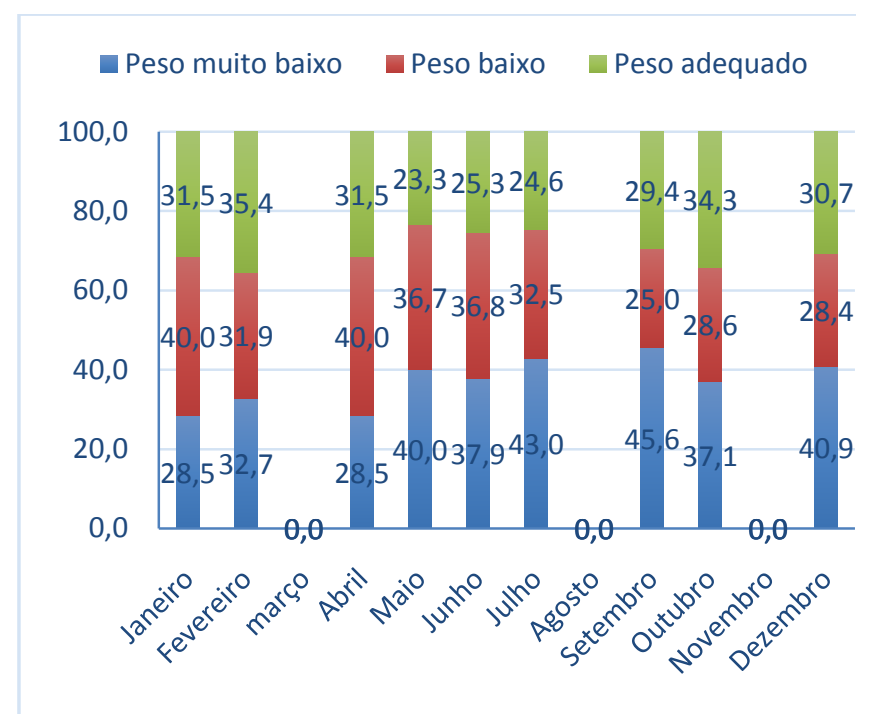

Fonte: Distrito Especial de Saúde Indígena-DSEY/ SESAI (2015).

Analisando a figura 3 podemos observar que, em janeiro de 2014 o percentual era de $28,5 \%$ para crianças com muito baixo peso e em dezembro de 2014, considerando a mesma faixa etária o percentual de crianças com muito baixo peso foi de 40,9\%; pode ser observado também uma redução de 18,4\% dos casos de crianças com baixo peso e de menos de $1 \%$ para crianças com peso adequado nos meses de janeiro em relação a dezembro.

Observe a figura 4 que apresenta o percentual médio do estado nutricional de crianças com 0 - 5 anos de idade, no polo base Haxiu, no DESY Yanomami, Roraima.
Figura 4 - Percentual médio do estado nutricional de crianças com 0 - 5 anos de idade, no polo base Haxiu, no DESY Yanomami, Roraima

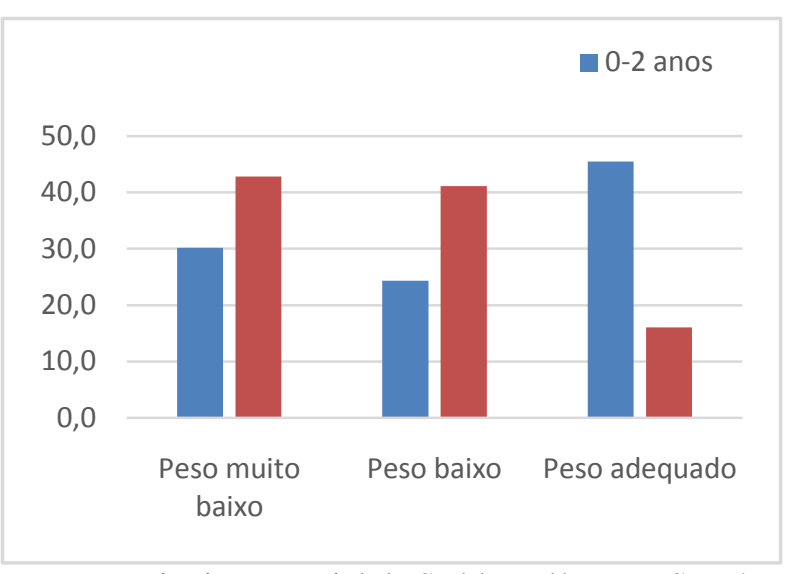

Fonte: Distrito Especial de Saúde Indígena-DSEY/ SESAI (2015).

Analisando a figura 4, verifica-se que o percentual médio de crianças com idade entre 0 - 2 anos com seu estado nutricional classificado como muito baixo peso é $12,6 \%$ inferior a crianças com idades entre 2-5 anos para o mesmo estado nutricional e $16,7 \%$ inferior para o estado nutricional classificado como baixo peso; verifica-se também quecrianças classificadas como peso adequado na faixa etária entre $2-5$ anos de idade, é de aproximadamente $30 \%$ superior neste estado nutricional em relação a crianças de $0-2$ anos de idade.

Observe a figura 5 que apresenta o percentual da faixa etária de crianças com $0-5$ anos de idade, no polo base Haxiu, no DESY Yanomami, Roraima. 
Figura 5 - Percentual da faixa etária de crianças com 0 -5 anos de idade, no polo base Haxiu, no DESY Yanomami, Roraima

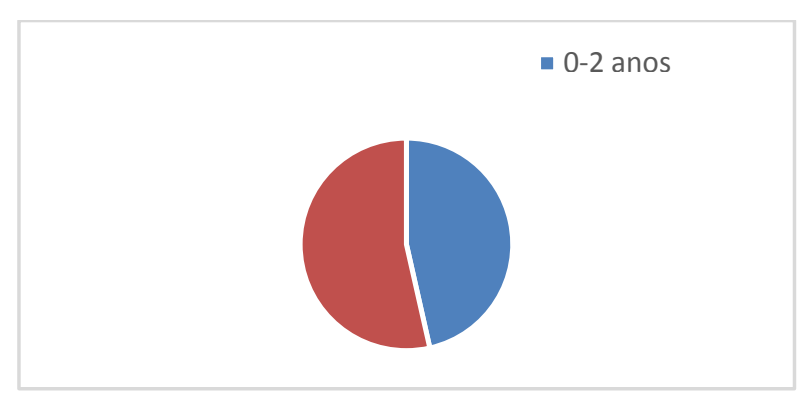

Fonte: Distrito Especial de Saúde Indígena-DSEY/ SESAI (2015).

Analisando a figura 5, verifica-se que o percentual de crianças com idade entre $2-5$ anos corresponde a $54 \%$ do total sendo aproximadamente $8 \%$ superior a crianças com faixa etária de 0 - 2 anos.Para o ano de 2014 a prevalência de crianças com idade entre 0 e 5 anos classificadas como "peso muito baixo" e "peso baixo" foi de 0,7 para o pólo base Haixiu.

\section{Discussão}

No Polo Base Haxiua desnutrição afetou mais as crianças com idades de 2 a 5 anos, levando em consideração o que os autores falam sobre os fatores de risco, podemos destacar que a comunidade faz parte das comunidades do DSEY Yanomami onde a maioria são povos isolados e que o acesso a um trabalho de educação em saúde pode estar comprometido e as condições em que as populações vivem pode estar influenciando o problema.

Entre os autores que estudaram a problemáticadestacam quepassado por muitas modificações e que tais mudanças afetam diversas dimensões da vida dos povos indígenas e os expõem a maiores riscos de desenvolvimento de problemas nutricionais, com todas as implicações que estes processos podem trazer à saúde,como discutiremos mais adiante (SESAI, 2015).

Mostra-se conforme dados do Fundo das Nações Unidas para a Infância (UNICEF) e do Programa das Nações Unidas para o Desenvolvimento (PNUD)' ainda é muito elevado também o Índice de desnutrição infantil em toda a região amazônica (SIEGEL, 1975).

O Inquérito Nacional de Saúde e Nutrição dos Povos Indígenas foi o primeiro grande estudo nacional envolvendo os povos indígenas do Brasil, realizado em 2008/2009 (CARNELO; PONTES, 2012).Os resultados apontaram para a emergência de excesso de peso e níveis pressóricos elevados nas mulheres, alta prevalência de anemia em mulheres e crianças, elevada proporção de crianças com déficit de crescimento, moderada prevalência de baixo peso para a idade e quase inexistência de déficits nutricionais pelo índice peso para estatura. Este fenômeno coincide com o que alguns autores vêm chamando de transição nutricional, relatado por estudos antropométricos realizados com populações pobres vítimas da fome endêmica. Uma defesa em relação a população indígena é que a aptidão ou não da mulher indígena exercer sua função materna, particularmente nos primeiros dois anos de vida, faixa etária em que a desnutrição é mais prevalente e pode incidir em suas formas graves, onde a criança 
é mais dependente da mãe para sobreviver e receber as melhores condições possíveis. Defende-se ainda que o fraco vínculo mãefilho seria o principal fator associado à desnutrição, pois a criança, não tendo todas as necessidades priorizadas e atendidas, fica exposta aos fatores adversos do meio ambiente, pois sem sua principal aliada na luta pela sobrevivência e por uma vida digna, poderá não ser alimentada adequadamente, não tendo dessa forma horários de lazer e sono respeitados (SILVA, 2009)'

Já na visão de Fávaro (2011), o baixo peso das crianças ao nascer exerce grande influência sobre seu crescimento e desenvolvimento, tornando-a assim mais vulnerável aos agravos de saúde. Outros fatores, como, moradia, mães jovens e com baixo grau de escolaridade mostraram uma elevação significante dos casos estudados.

A desnutrição infantil tem determinantes multicausais, com condicionantes biológicos e sociais que se relacionam com o atendimento (ou não) de suas necessidades básicas, como saúde, saneamento, educação e alimentação (YAMAMOTO, 2004).

Em um estudo onde foi observada as prevalências de desnutrição nas crianças Xavante mostraram-se excepcionalmente elevadas se comparadas àquelas para a população brasileira em geral, aproximandose das prevalências das regiões socioeconomicamente mais desfavorecidas no país (MASIERO et al, 2009)
São fatores como esse, que mostram influenciarem no aparecimento de doenças nutricionais, na cultura indígena, como já mencionado por outro autor, as mulheres indígenas se casam muito cedo, e consequentemente se tornam mães, o que pode desta forma dificultar o vínculo mãefilho já que a mesma pode não está preparada para tornar-se mãe, deixando assim a criança vulnerável e desprotegida contra os fatores externos, o que se pode evidenciar na quantidade de crianças com peso muito baixo e baixo peso nas comunidades abordadas no estudo.

\section{Referências}

BRASIL. Ministério da Saúde. Secretaria de Atenção à Saúde. Coordenação Geral da Política de Alimentação e Nutrição. Manual de atendimento da criança com desnutrição grave em nível hospitalar. Brasília: Ministério da Saúde, 2005. Ministério da Saúde. Manual Instrutivo para Implementação da Agenda para Intensificação da Atenção Nutricional à Desnutrição Infantil. 2014. Disponível em: <http://189.28.128.100/dab/docs/portaldab/pu blicacoes/capitulo_complem>. Acesso em: 15 mai. 2015.

CARNELO, L.; PONTES, L.A.Saúde indígena:Uma introdução ao tema.Brasília: UNESCO, 2012.

FÁVARO, Tatiana. Perfil nutricional da população indígena Xukuru de Ororubá, Pernambuco. 2011. Disponível em: $<$ http://indiosnonordeste.com.br/wpcontent/uploads/2012/08/Favaro-TR.TeseFinal.pdf>. Acesso em: 15 mai. 2015. 
INSTITUTO BRASILEIRO DE GEOGRAFIA E ESTATÍSTICA. Censo demográfico indígena [2010]. Disponível em:<http://www.ibge.gov.br/indigenas/indige na_censo2010.pdf $>$. Acesso em: 15 mai. 2015.

MASIERO, Kühlet al. Perfil Nutricional e Fatores Associados à Ocorrência de Desnutrição entre Crianças Indígenas Kaingáng da Terra Indígena de Mangueirinha, Paraná, Brasil.Cad. Saúde Pública, Rio de Janeiro, v. 25, n. 2, p. 409-420, fev. 2009. Disponível em:

<http://www.scielo.br/pdf/csp/v25n2/20>.Ace sso em: 15 mai. 2015.

PANTOJA, Lídia de Nazaré et al . Cobertura do Sistema de Vigilância Alimentar e Nutricional Indígena (SISVAN-I) e prevalência de desvios nutricionais em crianças Yanomami menores de 60 meses, Amazônia, Brasil. Rev. Bras. Saude Mater. Infant., Recife, v. 14, n. 1, p. 53-63, Mar. 2014. Disponível em: <http://www.scielo.br/scielo.php?script=sci_a rttext\&pid=S1519$38292014000100053 \& \operatorname{lng}=$ en $\& n r m=$ iso $>$. Acesso em: 15 mai. 2015.

PORTAL G1. Proporcionalmente Roraima tem maior populacao indígena do país.

2013. Disponível em: <http://www.G1.globo.com/rr/roraima/noticia /2013/04/proporcionalmente-roraima-temmaior-populacao-indigena-do-pais.html > . Acesso em: 15 mai. 2015.

SESAI.Secretaria Especial de Saúde Indígena. Departamento de Vigilância Epidemiológica. Boa Vista, Roraima 2015.

SIEGEL, S. Estatística não paramétrica: para as Ciências do Comportamento. São Paulo: McGraw-Hill; 1975.

SILVA, Hilton P. A Saúde Humana e a Amazônia no Século XXI: Reflexões Sobre os Objetivos do Milênio.Novos Cadernos NAEA, v. 9, n. 1, p. 77-94, jun. 2006.

Disponível em: <http://www.periodicos.ufpa.br/index.php/nc n/article/viewFile/58/130>. Acesso em: 15 mai. 2015.

WAITZBERG, Dan L.Nutrição Oral, Enteral e Parenteral na Prática Clínica.

São Paulo: Atheneu, 2009.

YAMAMOTO,Renato. Manual de Atenção à Saúde da Criança Indígena Brasileira. Brasília: [s.ed.], 2004. 